\title{
Physician wellbeing during the COVID-19 pandemic: an acute on chronic condition
}

\author{
Amanda A. Sandford ${ }^{1 凶}$ and John A. F. Zupancic ${ }^{1,2}$ \\ (c) The Author(s), under exclusive licence to the International Pediatric Research Foundation, Inc 2021
}

Pediatric Research (2022) 91:19-20; https://doi.org/10.1038/s41390-021-01750-w

Physicians benefit as members of a profession rooted in altruism and find joy, or compassion satisfaction, from helping those in need. Concurrently, by the very nature of this work, a physician bears witness to suffering, trauma, and loss of life. Over time, repeatedly engaging in intensive caregiving without adequate restorative opportunities can result in compassion fatigue, or a secondary "vicarious" traumatization. Caregivers experiencing compassion fatigue may lose their sense of meaning, become disengaged, irritable, isolated, and manifest with somatic complaints. $^{1}$

While physicians are at risk for compassion fatigue due to the caregiving nature of the profession, they may also experience professional burnout, described as increased emotional exhaustion, depersonalization, and a reduced sense of personal accomplishment. ${ }^{2}$ Among physicians, the prevalence of burnout is particularly high, with $49 \%$ of US physicians in 2016 reporting that they "often or always experience feelings of burnout." ${ }^{\prime 3}$ The condition is associated with a myriad of downstream personal and professional effects including decreased patient satisfaction, ${ }^{4}$ poorer physician mental health, ${ }^{5}$ and medical error. ${ }^{6}$ Many of the symptoms of burnout overlap with those of compassion fatigue.

While increased attention and resources have been directed toward healthcare worker burnout recently, physician wellbeing has remained in guarded condition. With the onset of COVID-19, increases in occupational hazards and personal stressors caused further disruption. Exposure to repeated loss of life, the threat of loss of life, fatigue, and isolation, as well as the loss of daily routine and grounding rituals, resulted in heightened mood and sleep disturbances, anxiety, and depression. ${ }^{7}$ Regardless of specialty, individuals were required to make sense of and respond to everchanging information. Challenges related to rationing resources, the conflicting duty to home and patients, and serving in crisis role capacities without adequate training created conditions predisposing to moral injury. ${ }^{8}$

Addressing burnout and compassion fatigue are critical elements to ensure long-term physician wellbeing and workforce resilience, paramount today given pandemic stressors. Kase et al. make an important contribution toward advancing that goal by comparing key measures related to physician wellbeing among pediatric subspecialists in neonatology, hematology/oncology, palliative care, emergency medicine, and critical care prior to (2016-2019) and early in the pandemic (June to July 2020). The authors hypothesized that compassion fatigue and burnout scores would be higher during the early pandemic. They employed the Modified Compassion Fatigue and Satisfaction Self Care Test for Helpers, ${ }^{1}$ a previously validated instrument comprising three subdomains: Compassion Fatigue, Burnout, and Compassion Satisfaction. Among 7840 subspecialists surveyed, response rates averaged $26 \%$ and varied by specialty.

Results included higher compassion fatigue scores during the prepandemic baseline for critical care physicians compared to neonatologists and hematologist/oncologists, and higher critical care burnout scores compared to palliative care physicians. Similarly, prepandemic critical care and emergency physicians had lower compassion satisfaction than neonatologists and palliative care specialists. Not all specialties seem to have reacted to the pressures of the pandemic in the same manner. Early pandemic compassion fatigue scores in neonatology and hematology/oncology increased, while critical care and emergency medicine scores decreased. These divergent responses may explain the unexpected absence of a difference on average in pre- and early pandemic compassion fatigue, burnout, and compassion satisfaction and suggest the complex and multifactorial nature of these domains. The authors also acknowledge the potential impact of various study limitations, including the response rate, potential for nonresponse bias, and a homogenous study population.

In the analysis of factors associated with compassion fatigue, putting oneself at "increased risk through work," burnout, and distress about mental health were statistically significant. Conversely, compassion satisfaction and feeling valued by the institution for one's contributions during the COVID-19 crisis mitigated compassion fatigue.

Kase et al.'s report leverages the stress of a global health crisis to highlight three more general issues in our community. First, it furthers our understanding of compassion fatigue and its prevalence. Although more widely studied in nurses, there is limited literature on physician impact. A systematic review of studies set in intensive care units showed wide variability, with heightened levels of compassion fatigue or secondary stress in up to $40 \%$ of participants. ${ }^{9}$ Broader studies of prevalence confirming the generalizability of these findings and exploring potential contributing factors are indicated.

Second, the authors demonstrate that increasing compassion satisfaction and feeling valued by an organization are protective. While it is important to implement measures to address compassion

\footnotetext{
${ }^{1}$ Department of Neonatology, Beth Israel Deaconess Medical Center, Boston, MA, USA. ${ }^{2}$ Division of Newborn Medicine, Harvard Medical School, Boston, MA, USA. email: asandfor@bidmc.harvard.edu
} 
fatigue, ample efforts should be directed toward fostering compassion satisfaction. Recognizing and appreciating one's efforts contributes to a healthy workplace environment. Transformation of the medical culture by integrating practices that emphasize the positive aspects of caregiving can reframe healthcare workers' experiences in a positive manner. For example, a simple intervention asking participants to identify "three good things" daily for 15 days resulted in improvements in emotional exhaustion, depression symptoms, and happiness up to 12 months after the intervention. ${ }^{10}$

Finally, although the pandemic heightened awareness and urgency regarding the stress and suffering of physicians, the authors' work underscores that compassion fatigue and burnout were pervasive long before COVID-19. Approaching these issues as occupational hazards rather than acute pathology may enable physicians to embrace the commonality of these conditions and the tolls of a profession that bears witness to suffering.

Physician wellbeing, the antithesis of compassion fatigue and burnout, is influenced by an amalgamation of organizational culture, efficiency of practice, and personal resilience. ${ }^{11}$ Reversing compassion fatigue and burnout trends will require a multifaceted approach with both individual and organizational strategies. With an increased attentiveness to our occupational stress, we can create a meaningful rather than traumatic narrative. ${ }^{12}$ Kase et al.'s report encourages us to take advantage of this moment to emphasize physician wellbeing and to provide frontline healthcare workers with the mental health support needed to sustain them through the pandemic and beyond.

\section{REFERENCES}

1. Figley, C. R. Compassion Fatigue (Taylor \& Francis, 1995).

2. Maslach, C. \& Jackson, S. E. The measurement of experienced burnout. J. Organ. Behav. 2, 99-113 (1981).

3. 2016 Survey of America's Physicians. Survey conducted on behalf of the Physicians Foundation by Merritt Hawkins. The Physicians Foundation www. physiciansfoundation.org (2016).

4. Halbesleben, J. R. B. \& Rathert, C. Linking physician burnout and patient outcomes: exploring the dyadic relationship between physicians and patients. Health Care Manag. Rev. 33, 29-39 (2008).
5. McFarland, D. C., Hulbocky, F. \& Riba, M. Update on addressing mental health and burnout in physicians: What is the role for psychiatry? Curr. Psychiatry Rep. 21, 108 (2019).

6. Tawfik, D. S. et al. Physician burnout, well-being, and work unit safety grades in relationship to reported medical errors. Mayo Clin. Proc. 93, 1571-1580 (2018).

7. Pappa, S. et al. Prevalence of depression, anxiety, and insomnia among healthcare workers during the COVID-19 pandemic: a systematic review and metaanalysis. Brain Behav. Immun. 88, 901-907 (2020).

8. Greenberg, N. Mental health of health-care workers in the COVID-19 era. Nat. Rev. Nephrol. 16, 425-426 (2020).

9. van Mol, M. M., Kompanje, E. J., Benoit, D. D., Bakker, J. \& Nijkamp, M. D. The prevalence of compassion fatigue and burnout among healthcare professionals in intensive care units: a systematic review. PLOS ONE 10, e1-e22 (2015).

10. Sexton, J. B. \& Adair, K. C. Forty-five good things: a prospective pilot study of the Three Good Things well-being intervention in the USA for healthcare worker emotional exhaustion, depression, work-life balance and happiness. BMJ Open 9, e022695 (2019).

11. Bohman, B. et al. Physician well-being: the reciprocity of practice efficiency, culture of wellness, and personal resilience. NEJM Catalyst http://catalyst.Nejm. Org/physician-well-being-efficiency-wellness-resilience/ (2017).

12. Watson, P. Caring for yourself and others during the COVID-19 pandemic [Webinar]. Schwartz Center for Compassion in Action https://www. theschwartzcenter.org/webinar/caring-for-yourself-others-during-the-covid-19pandemic-managing-healthcare-workers-stress/ (2020).

\section{COMPETING INTERESTS}

The authors declare no competing interests.

\section{ADDITIONAL INFORMATION}

Correspondence and requests for materials should be addressed to Amanda A. Sandford.

Reprints and permission information is available at http://www.nature.com/ reprints

Publisher's note Springer Nature remains neutral with regard to jurisdictional claims in published maps and institutional affiliations. 